\title{
Corn Drying Process Variables Screening and Its Moisture Content Forecast
}

\author{
Kefei Wang ${ }^{1, a}$, Ming Lu ${ }^{1, b}$ and Hongdi Ke ${ }^{1, c^{*}}$ \\ 1 Department of Technology,Jilin Business and Technology College,Changchun 130507,China \\ àwangkefei888@sina.com, b975579008@qq.com , c13661033@qq.com
}

\section{Keywords: Moisture Content; Corn Drying; BP Neural Network; Variables Screening}

\begin{abstract}
This study is based on BP neural network technology, through the MATLAB programmed processing, analyzed the factors which influenced the corn moisture content.Finally,through the MATLAB ,designed a method of online moisture content of corn. Through the comparison of predictive value and the true value of the corn. The prediction accuracy of this method could run up to 95\% under the same batches. This method provide a reliable theoretical basis for the online determination of moisture content of corn.
\end{abstract}

\section{Introduction}

China is the world's largest grain producer and consumer, the total production of about 500 million tons of grain. According to the survey due to grain drying technology, often due to lack of timely grain drying, or drying failed, causing losses of up to $15 \%$, far more than $5 \%$ of the standard provisions of the FAO[1-2].

Grain drying chamber agricultural production process of the key aspects. Select the neural network input There are many ways, from the basic idea is significant as the effect of independent variables selected into the neural network model, the effect is not significant independent variables excluded.

we will combine BP neural network average impact value (MIV, Mean Impact Value) method to illustrate how to use neural network screening variables, find a greater impact on the results and entries, and then use neural networks to achieve variable selection. This screening method is applied to the corn drying process variables, with the variables that have been screened on the moisture content during drying of maize modeling.

\section{Corn Drying Process Variables Based on BP Neural Network Screening}

Corn Drying Process. A drying section has 6 cooling segments. Independent factors affected are 14, A entire dry process is near 8 hours, each section takes about 2 hours.

Corn drying process variables are, S 1 and T 1, respectively at the outlet of the dryer the moisture content and temperature of maize, S2 and T2, respectively, at the outlet of a dry corn moisture and sixth temperature, T 3 at the outlet of a dry four maize temperature, T 4 at the outlet of a dry corn Sec temperature, $\mathrm{T} 5$ for the unprocessed temperature, $\mathrm{T} 6, \mathrm{~T} 7, \mathrm{~T} 8$, and a hot air temperature T11, T9, and $\mathrm{T} 10$ for the exhaust gas temperature, $\mathrm{T} 12$ is the ambient temperature, $\mathrm{n}$ is row grain motor speed.

Corn Drying Process Variables based on BP Neural Network The full name of BP neural network algorithm based on error back propagation neural network, its topology shown in Fig. 1.

BP Neural network has 3 layers: the input layer, intermediate layer (also called the hidden layer) and output layer[3,4]. Each input layer neurons responsible for receiving input information from the outside world, and passed to the detection of each neuron, the middle layer is the internal information processing layer, an intermediate layer can be designed as a single hidden layer or hidden layer structure; finally passed to a hidden layer information for each output layer neuron, after further processing, to complete a forward propagation learning process, outputs from the output layer to the outside output information processing results. 
Algorithm for the data stream prior to the calculation of (forward propagation) and the back-propagation of error signals constituting the two processes. When the forward propagation, propagation direction of the input layer $\rightarrow$ hidden layer $\rightarrow$ output layer[4,5,9,10], each neuron state only affects the next layer neurons. If the output layer are not expected output, then turned back propagation process error signal. By alternating these two processes were performed in the weight vector of the error function gradient space drops strategy, dynamic iterative search a weight vector, the network error function reached a minimum value, thereby completing the information extraction and memory processes.

MIV is considered the best indicator variables evaluated questioned correlation in a neural network, but also to solve such problems and create new ideas.

In this paper, the importance of selecting the size of MIV as an evaluation index of each independent variable on the dependent variable impacts. MIV is used to determine the input neurons to the output neurons affect the size of an index, the symbol representing the relevant direction, size represents the absolute value of the relative importance of the impact[3-4]. The calculation process: After the termination of the network training, the training sample $\mathrm{P}$ features in each independent variable on the basis of its original value, respectively, plus / minus $10 \%$ constitute a new two training samples P1 and $\mathrm{P} 2$, P1 and P2, respectively, as the simulation samples have been built using network simulation, two simulation results $\mathrm{A} 1$ and $\mathrm{A} 2, \mathrm{~A} 1$ and $\mathrm{A} 2$ calculated difference, namely changes on changes in the value (IV) after the output generated by the argument, and finally by observing IV the average number of cases should come to the argument for network output variables ---- MIV. Calculated in accordance with the above procedure followed MIV value of each independent variable, according to MIV definitely worth the final size of each variable ordering, get their own network output variable relative importance of ranking table to determine the extent of the impact on network input feature results, namely achieve a variable selection.

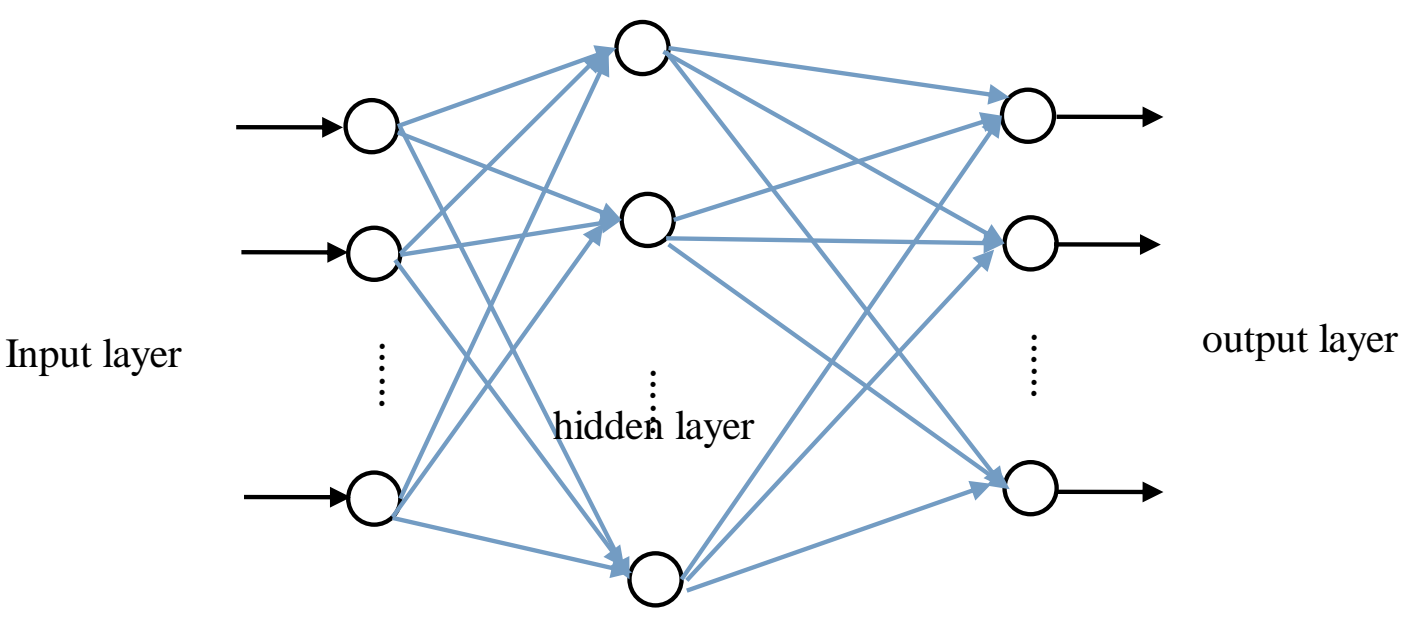

Figure 1 BP neural network structure diagram

Variable Selection to Achieve. Select corn drying process 200, each taking the test time, i.e. $2 \mathrm{~h}, 4 \mathrm{~h}$, $6 \mathrm{~h}, 8 \mathrm{~h}$, a total of 800 sets of data, S 1 and T 1, respectively at the outlet of the dryer and the temperature of the water content of maize, S2 and T2, respectively, sixth at the exit for dry corn moisture content and temperature, $\mathrm{T} 3$ for four dry corn at the exit temperature, T 4, Sec dry corn temperature at the outlet, $\mathrm{T} 5$ as raw grain temperature, $\mathrm{T} 6, \mathrm{~T} 7, \mathrm{~T} 8$ and $\mathrm{T} 11$ hot air temperature, T9, and T10 for the exhaust gas temperature, $\mathrm{T} 12$ is the ambient temperature, $\mathrm{n}$ is the motor speed grain discharge. $\mathrm{S} 1$ as the dependent variable, the other variables as independent variables, MIV method of screening and the results of the main variables of the network affected. Shown as Fig. 2: 


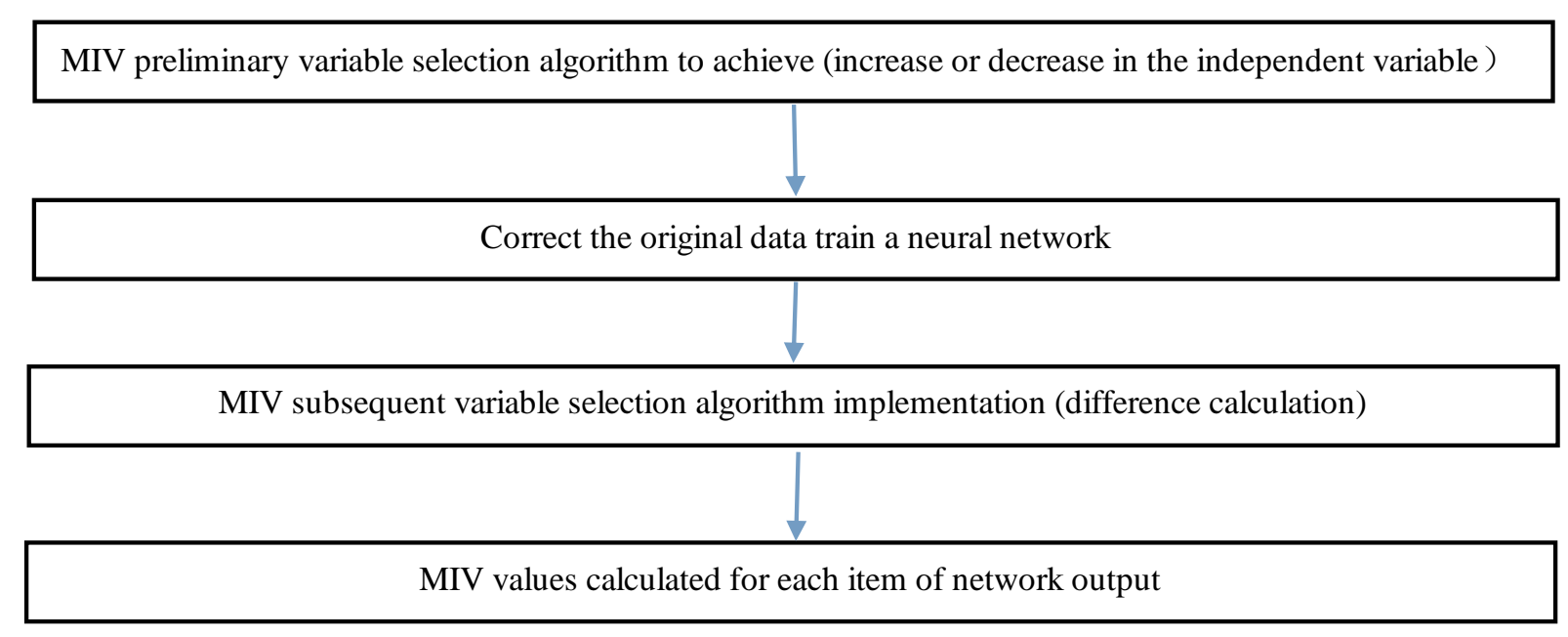

Figure 2. MIV flow chart

MATLAB Simulation.MIV the network output value is calculated in the following table:

Table1: MIV compare

\begin{tabular}{|c|c|}
\hline varialbles & MIV \\
\hline T1 & 1.2896 \\
\hline S2 & 1.2664 \\
\hline T2 & 1.2320 \\
\hline T3 & 1.0211 \\
\hline T4 & 0.9562 \\
\hline T5 & 0.9567 \\
\hline T6 & 0.6012 \\
\hline T7 & 0.5734 \\
\hline T8 & 0.5673 \\
\hline T9 & 0.5621 \\
\hline T10 & 0.0985 \\
\hline T11 & 0.1021 \\
\hline T12 & 0.1042 \\
\hline $\mathrm{n}$ & 0.9781 \\
\hline
\end{tabular}

\section{Based on BP Neural Network Training and Regression Forecast}

Therefore, we use a three improved BP neural network algorithm based feed forward BP network prediction model to establish a moisture content of grain dryer exit. According to Table 1 and to remove the output of a minimum T10, T11, T12. Determining the input variables 11.

Requirements hidden layer nodes to determine the problem, the number of input and output units have a direct relationship, hidden layer nodes will cause the network to learn too little too low or accuracy of the network does not converge, but too much will cause the network learning time too long, fault tolerance is poor, does not recognize without learning samples. Selected based on experience here first hidden layer nodes, and then observed network performance, increase or decrease the number of nodes in the hidden layer based on the observed results, a comprehensive comparison of the effects of each hidden layer nodes to predict the performance of the selected value, making the final choice network prediction error most hours of hidden layer nodes. After repeated experiments, select the "11-16-1" at the exit end of the dryer moisture corn forecast network.

Select the logarithmic transfer function S-type (Log-Sigmoid) transfer function,

$$
f(x)=\frac{1}{1+e^{-x}}
$$


Corn moisture content prediction model based networks were expected output S1, the output unit of the output layer $\hat{S} 1$, then the cost function fitting error of corn moisture content were forecasting network

$$
\mathrm{E}_{\mathrm{S} 1}=\frac{1}{2} \sum_{k=1}^{P}(S 1-\hat{S} 1)^{2}
$$

Among them, $\mathrm{P}$ is the number of training samples.

Using the quasi-Newton method to train the network[4,5,6], adjust the network connection weights to make the minimum cost function, concrete steps are:

(1) given initial point $x_{1} \in R^{n}$ and positive definite matrix $B_{1} \in R^{n \times n}, k=1$;

(2) caculate $g_{1}=\nabla E\left(x_{1}\right)$, if $g_{1}=0$, then stop[7,8], else continue;

(3) caculate $d_{k}=-B_{k}^{-1} g_{k}$, obtained search direction;

(4) along the search direction, according to Wolfe search model

$$
\begin{aligned}
& E\left(x_{k}+\alpha_{k} d_{k}\right)-E\left(x_{k}\right) \leq \delta_{1} \alpha_{k} d_{k}^{T} g_{k} \\
& d_{k}^{T} \nabla E\left(x_{k}+\alpha_{k} d_{k}\right) \geq \delta_{2} d_{k}^{T} g_{k}
\end{aligned}
$$

Calculated step, wherein, $\delta_{1}$ and $\delta_{2}$ is constant, and $\delta_{1} \in(0,1), \delta_{2} \in(0,1), \delta_{1} \leq \delta_{2}$; (5) orders $x_{k+1}=x_{k}+\alpha_{k} d_{k}$, caculate $g_{k+1}=\nabla E\left(x_{k+1}\right)$, if $g_{k+1}=0$, then stop, else continue;

(6) orders

$$
B_{k+1}=B_{k}-\frac{B_{k} s_{k} s_{k}^{T} B_{k}}{s_{k}^{T} B_{k} s_{k}}+\frac{y_{k} y_{k}^{T}}{s_{k}^{T} y_{k}}
$$

wherein, $s_{k}=\alpha_{k} d_{k}, \quad y_{k}=g_{k+1}-g_{k}$;

(7) orders $k=k+1$, goto(3).

\section{Conclusion}

5000 sample data set classification, 2500 set as the training set of sample data, another 2500 set as the test sample data sets, with training set for BP training available predictive model, and then the resulting model to predict the test set. After fitting effect MSE $=0.0031472$, squared correlation coefficient $\mathrm{R}=$ $99.3124 \%$, to achieve the desired prediction. The prediction accuracy of this method could run up to $95 \%$ under the same batches.

The moisture content of corn drying process forecast for energy corn drying process, as well as storage of corn has a very important role, corn drying process variables screening and it's moisture content forecast article describes the moisture content in corn drying in the forecast regression. Application example shows that predictions is reliable in practice, can be widely extended. 


\section{Acknowledgements}

The research conducted in this paper have been supported by the fund of Important Foundation of Jilin Provincial Education department.(2013395) and JiLin Business and Technology doctor fund(No.201626).

\section{References}

[1] Li Jiang bo,Su Yi nan,Rao Xiu qin:Packing and Food Machinery,Vol.1(2010).No.6,P1-4.

[2] K.Z. Tan: Research on Quality of Soybean Using Hyperspecatral Imaging and Machine Vision Technquei(Ph.D.,Electrical and Information College, China 2014), p.65.

[3] Zhu Xu dong:Grain Distribution Technology,Vol.1(2011).No.5,P23-25.

[4] H.D. Ke: The Online Multiple Parameter Analysisi Technology of Smelt and End-point Control Tactics Research and Realize (Ph.D., Changchun Institute of Optics,Fine Mechanics and Physics Chines Academy of Science, China 2010), p.69.

[5] Cai You jie,Wu Zhi dong,Wang Xue feng,Bao Li and Zhang Jin feng:Food and Machinery,Vol.33(2017).No.2,P85-89.

[6] Li Su lei,Gao Hong ju,Feng lei,Li Li and Liang Dong:Transducer and Microsystem Technologies,Vol.33(2014).No.5,P39-44.

[7] Sun Yong hai,He Xiao Ping and Sun Yu:Journal of Jilin University(Engineering and Technology Edition),Vol.36(2015).No.5,P1177-1184.

[8] Atlas Khan: Opimizations of and by Neural Networks(Ph.D.,Dalin University of Technology, China 2013), p.1.

[9] H.Liu,J.Han: MATLAB R2012a Entirely Self-taught(Pubulishing House of Industry, China 2014).

[10] Information on http://www.ilovematlab.cn/forum.php. 